\begin{tabular}{|c|l|}
\hline Title & Investigation on multi-core fibers with large A eff and low micro bending loss \\
\hline Author(s) & Imamura, Katsunori; Tsuchida, Y ukihiro; Mukasa, Kazunori; Sugizaki, Ry uichi; Saitoh, Kunimasa; Koshiba, Masanori \\
\hline Citation & $\begin{array}{l}\text { Optics Express, 19(11), 10595-10603 } \\
\text { https:/doi.org/10.1364/0E.19.010595 }\end{array}$ \\
\hline Issue Date & 2011-05-23 \\
\hline Doc URL & http://hdl.handle.net/2115/45610 \\
\hline Rights & @2011 Optical Society of A merica \\
\hline Type & article \\
\hline File Information & OE19-11_10595-10603.pdf \\
\hline
\end{tabular}

Instructions for use 


\title{
Investigation on multi-core fibers with large Aeff and low micro bending loss
}

\author{
Katsunori Imamura, ${ }^{1,}{ }^{*}$ Yukihiro Tsuchida, ${ }^{1}$ Kazunori Mukasa, ${ }^{1}$ Ryuichi Sugizaki, ${ }^{1}$ \\ Kunimasa Saitoh, ${ }^{2}$ and Masanori Koshiba ${ }^{2}$ \\ ${ }^{\text {I}}$ Fitel Photonics laboratory, Furukawa Electric Co., Ltd., 6, Yawata-kaigandori, Ichihara, Chiba, 290-8555, Japan \\ ${ }^{2}$ Division of Media and Network Technologies, Hokkaido University, Sapporo, 060-0814, Japan \\ *imamura.katsunori@furukawa.co.jp
}

\begin{abstract}
To realize large effective area (Aeff) multi-core fibers (MCFs), the design to suppress the cross-talk and the influence of the cladding diameter on the micro bending loss were investigated. As a result, the MCFs with large Aeff over $100 \mu \mathrm{m}^{2}$ and low micro bending loss were successfully fabricated. The results indicate the importance of fiber design to realize large Aeff MCFs including fiber diameters, which largely affect the micro bending loss property. Additionally, MCF with large Aeff, low attenuation loss and suppressed cross-talk was successfully realized by optimizing the fiber design. The cross-talk properties could be estimated by the simulation based on the coupling power theory taking the influences of the longitudinal fluctuation of core diameter into account.
\end{abstract}

(C)2011 Optical Society of America

OCIS codes: (060.4005) Microstructured fibers; (060.2280) Fiber design and fabrication.

\section{References and links}

1. S. Inao, T. Sato, S. Sentsui, T. Kuroha, and Y. Nishimura, "Multicore optical fiber," in Optical Fiber Communication, 1979 OSA Technical Digest Series (Optical Society of America, 1979), paper WB1. http://www.opticsinfobase.org/abstract.cfm? URI=OFC-1979-WB1

2. M. J. Gander, W. N. MacPherson, R. McBride, J. D. C. Jones, L. Zhang, I. Bennion, P. M. Blanchard, J. G. Burnett, and A. H. Greenaway, "Bend measurement using Bragg gratings in multicore fibre," Electron. Lett. 36(2), 120-121 (2000).

3. Y. Huo, P. K. Cheo, and G. G. King, "Fundamental mode operation of a 19-core phase-locked Yb-doped fiber amplifier," Opt. Express 12(25), 6230-6239 (2004), http://www.opticsinfobase.org/oe/abstract.cfm?uri=oe-1225-6230.

4. N. N. Elkin, A. P. Napartovich, V. N. Troshchieva, and D. V. Vysotsky, "Diffraction modeling of the multicore fiber amplifier," J. Lightwave Technol. 25(10), 3072-3077 (2007).

5. T. Morioka, "New generation optical infrastructure technologies: 'EXAT Initiative' towards 2020 and beyond," in Proceedings of OptoElectronics and Communications Conference (2009), paper FT4.

6. M. Koshiba, K. Saitoh, and Y. Kokubun, "Heterogeneous multi-core fibers: proposal and design principle," IEICE Electron. Express 6(2), 98-103 (2009).

7. K. Imamura, K. Mukasa, and T. Yagi, "Multi-core holey fibers for the long-distance (>100 km) ultra large capacity transmission," in Proceedings of Optical Fiber Communications Conference (2009), paper OTuC3. http://www.opticsinfobase.org/abstract.cfm? URI=OFC-2009-OTuC3

8. C. Sethumadhavan, X. Liu, B. Zhu, and D. W. Peckham, "Transmission of a 1.2-Tb/s 24-carrier no-guardinterval coherent OFDM superchannel over 7200-km of ultra-large-area fiber," in Proceedings of European Conference and Exhibition on Optical Communications (2009), paper PD2.6.

9. G. Charlet, M. Salsi, P. Tran, M. Bertolini, H. Mardoyan, J. Renaudier, O. Bertran-Pardo, and S. Bigo, "72x100Gb/s Transmission over Transoceanic Distance, Using Large Effective Area Fiber, Hybrid RamanErbium Amplification and Coherent Detection," in Proceedings of Optical Fiber Communications Conference (2009), paper PDPB6. http://www.opticsinfobase.org/abstract.cfm?URI=OFC-2009-PDPB6

10. K. Takenaga, S. Tanigawa, N. Guan, S. Matsuo, K. Saitoh, and M. Koshiba, "Reduction of Crosstalk by QuasiHomogeneous Solid Multi-Core Fiber," in Proceedings of Optical Fiber Communications Conference (2010), paper OWK7. http://www.opticsinfobase.org/abstract.cfm?URI=OFC-2010-OWK7

11. K. Nakajima, M. Ohashi, and M. Tateda, "Chromatic dispersion distribution measurement along a single-mode optical fiber,” J. Lightwave Technol. 15(7), 1095-1101 (1997).

\section{Introduction}

Multi-core fibers (MCFs) have a large potential to increase the transmission capacity drastically by space division multiplexing (SDM). Though this concept had already been 
suggested 30 years ago [1], interests had been concentrated on other regions than the long distance transmission, e.g. sensors, fiber lasers, et al. [2-4]. Recently, it has been pointed out that the transmission capacity will be exhausted in the near future [5], and the new technologies should be needed for the transmission fibers. Since then, MCFs for the long distance transmission have rapidly been paid great attention as one of the attractive candidates for that new transmission line and the developments of solid and holey types of MCFs have recently been reported [6,7]. On the other hand, the ways to increase the capacity per one fiber have also been intensely studied [8,9]. In these reports, not only the improved modulation formats but also the enlarged effective area (Aeff) of transmission fibers was greatly contributed to increase the transmission capacity. However, the reported MCFs have only small Aeff compared to conventional single mode fiber (SMF). So, it is expected that the MCFs with large Aeff must have a great impact on the future optical transmission systems.

For the design of the large Aeff MCFs, the interference between cores and the micro bending loss are the most significant problems, which needs to be addressed. In this paper, we studied the design to suppress the increase of the interference between cores in case of the large Aeff fibers and found out that the Aeff can be enlarged without increasing the cross-talk between cores by keeping the macro bending loss constantly. The influence of the cladding diameter on the micro bending loss was investigated and the solid MCFs with large Aeff over $100 \mu \mathrm{m}^{2}$ and low micro bending loss were actually fabricated by means of the adequate design with the sufficient cladding thickness. The low cross-talk properties were successfully realized by the design optimization and could be estimated by the simulation based on the coupled power theory taking the longitudinal fluctuation of core diameter into account.

\section{Micro bending losses of multi-core fibers with different cladding diameters}

\subsection{Design of multi-core fibers with large Aeff and low cross-talk}

At first, we investigated the design strategy required for enlargement of Aeff of MCFs. As the Aeff is increased, we need to pay much attention to the increase of interference between cores. We thoroughly investigated the relation between Aeff at $1550 \mathrm{~nm}$, macro bending loss at 20 $\mathrm{mm}$ diameter at $1550 \mathrm{~nm}$ and core pitch required for the $100 \mathrm{~km}$ transmission. The target cross-talk was set to $-30 \mathrm{~dB}$ at $1550 \mathrm{~nm}$ after $100 \mathrm{~km}$ transmission. As the result, we found out that the core pitch can be maintained even when Aeff was enlarged, if we set the macro bending loss conditions to be the same (Fig. 1). By using this concept, we can design the large Aeff solid MCFs by shifting the cut-off wavelength $(\lambda \mathrm{c})$ and keeping the macro bending loss constant without increase of cross-talk between cores.

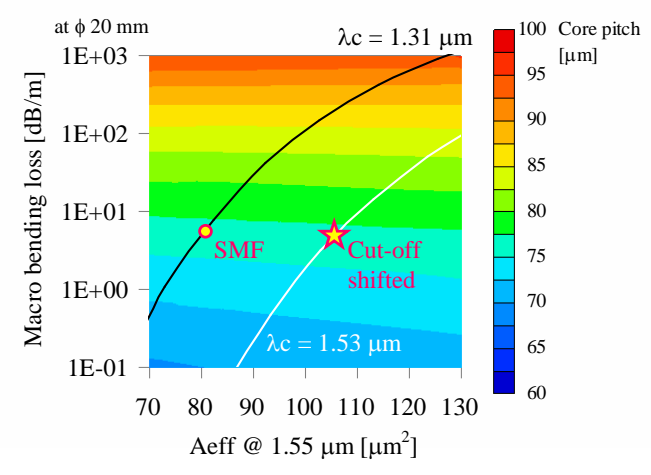

Fig. 1. Relationship between Aeff, macro bending loss and core pitch required to realize 100 $\mathrm{km}$ transmission with low cross-talk.

On the other hand, a high core density packaging technique is proposed by Koshiba et al. [6]. It was reported that the power-conversion efficiency can be decreased drastically by combining some cores with slightly different refractive index in one fiber. However, the 
reported Aeff was same as or smaller than conventional SMF. This method can be applied for the large Aeff MCFs in this study, so we investigated the effect of this technique on our large Aeff design and obtained the relation between core diameter and maximum cross-talk as shown in Fig. 2. The effective refractive index was changed just by changing the core diameter a little keeping the core $\Delta(=0.31 \%)$ for a simplification of fabrication process. The core pitch was set to $42 \mu \mathrm{m}$, which corresponds to the pitch of $75 \mu \mathrm{m}$ in the case of identical cores. As a result, by changing the core diameter just by $0.2 \mu \mathrm{m}$, the cross-talk between neighboring cores (different cores) can be decreased under $-60 \mathrm{~dB}$. We optimized the core diameter to keep the cable cut-off wavelength $(\lambda \mathrm{cc})$ below $1530 \mathrm{~nm}$ and macro bending loss below $5 \mathrm{~dB} / \mathrm{m}$ even when the core diameters are changed to $+/-0.2 \mu \mathrm{m}$ (Table 1$)$. From these calculations, it was confirmed that the solid MCFs with Aeff as $100 \mu \mathrm{m}^{2}$ can be realized with high core density.

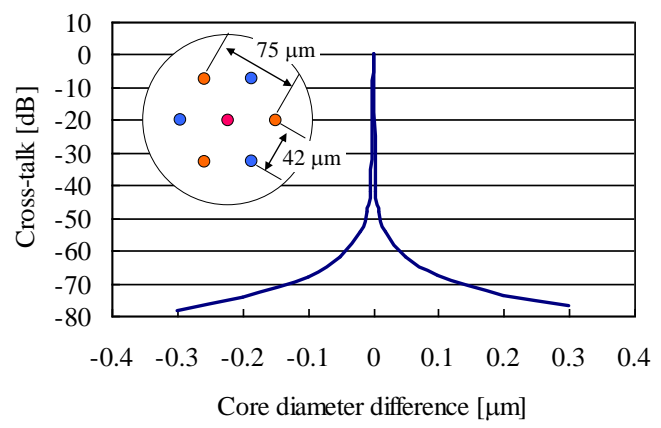

Fig. 2. Cross-talk between neighboring cores (@1550 nm).

Table 1. Design Properties of Each Core

\begin{tabular}{|c|c|c|c|}
\hline Core diameter & \multicolumn{1}{|c|}{ Aeff } & $\lambda \mathrm{cc}$ & Bend loss \\
\hline$\mu \mathrm{m}$ & \multicolumn{1}{|c|}{$\mu \mathrm{m}^{2}$} & $\mathrm{~nm}$ & $\mathrm{~dB} / \mathrm{m}$ \\
\hline 10.2 & 100 & 1460 & 3.2 \\
\hline 10.4 & 102 & 1490 & 2.3 \\
\hline 10.6 & 104 & 1520 & 1.6 \\
\hline
\end{tabular}

\subsection{Examination of the influence of the cladding diameter on micro bending loss properties}

Based on the simulation results described in section 2.1, the solid MCFs, which have high productivities, were actually fabricated by means of stack \& draw method. Because the outer cores tend to be close to the cladding edge, the micro bending losses can be considered as one issue of the MCFs. So, we investigated the influence of the cladding diameter on micro bending loss properties. Two fibers with different cladding diameters (Fiber \#1: $141 \mu \mathrm{m}$, Fiber $\# 2: 215 \mu \mathrm{m}$ ) were fabricated to compare their micro bending loss properties. The cross sections of designed and fabricated fibers are shown in Figs. 3 and 4, respectively. The core pitch was $40 \mu \mathrm{m}$, which is almost same as the design, and the coating thickness were set to be equal for each fiber to keep the same influence of coating. To recognize the core arrangement, 3 air holes were arranged as markers at the region where they would not influence the optical properties. 


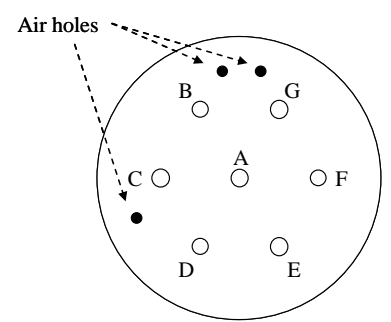

Fig. 3. Cross section of designed fiber with air holes as markers.

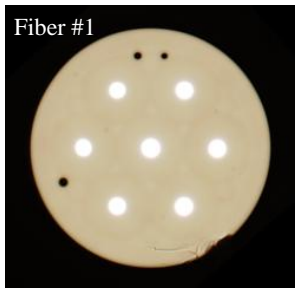

(a)

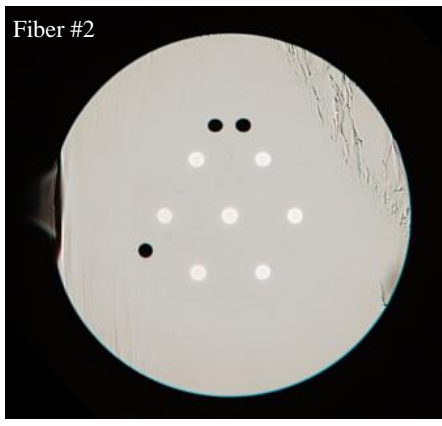

(b)

Fig. 4. Cross sections of fabricated fibers (a) Fiber \#1: $141 \mu \mathrm{m}$, (b) Fiber \#2: $215 \mu \mathrm{m}$.

Optical properties of two fibers are shown in Table 2. Because there are 3 kinds of cores, center core (core A) and other two neighboring cores (B, C) were measured. As shown in the attenuation loss spectra (Fig. 5), loss increase in the longer wavelength region were observed in case of fiber with small cladding diameter (Fiber \#1), especially in outer cores (B, C). On the other hand, in case of Fiber \#2 with large cladding diameter, loss increases at longer wavelength were successfully suppressed in each core. As the result, the effect of large cladding diameter for the reduction of micro bending losses was clearly confirmed.

Table 2. Optical Properties of Fiber \#1 and Fiber \#2 (@1550 nm)

\begin{tabular}{|c|l|l|l|l|l|l|l|}
\hline Fiber & \multirow{2}{*}{$\begin{array}{c}\text { Core } \\
\text { No. }\end{array}$} & Loss & Dispersion & D. Slope & Aeff & $\lambda \mathrm{cc}$ & \multicolumn{1}{c|}{ Bend loss * } \\
\cline { 3 - 8 } & $\mathrm{dB} / \mathrm{km}$ & $\mathrm{ps} / \mathrm{nm} / \mathrm{km}$ & $\mathrm{ps} / \mathrm{nm}^{2} / \mathrm{km}$ & $\mu \mathrm{m}^{2}$ & $\mathrm{~nm}$ & \multicolumn{1}{c|}{$\mathrm{dB} / \mathrm{m}$} \\
\hline Fiber \#1 & $\mathrm{A}$ & 0.254 & 19.0 & 0.062 & 110 & 1367 & 10.1 \\
\cline { 2 - 8 } & $\mathrm{B}$ & 0.444 & 18.1 & 0.060 & 103 & 1337 & 16.7 \\
\cline { 2 - 8 } & $\mathrm{C}$ & 0.398 & 18.6 & 0.060 & 106 & 1357 & 7.1 \\
\hline Fiber \#2 & $\mathrm{A}$ & 0.205 & 19.1 & 0.062 & 101 & 1378 & 4.4 \\
\cline { 2 - 8 } & $\mathrm{B}$ & 0.273 & 18.6 & 0.060 & 103 & 1367 & 7.2 \\
\cline { 2 - 8 } & $\mathrm{C}$ & 0.270 & 18.4 & 0.061 & 99 & 1357 & 13.3 \\
\hline
\end{tabular}

* Macro bending loss at $20 \mathrm{~mm}$ diameter. 


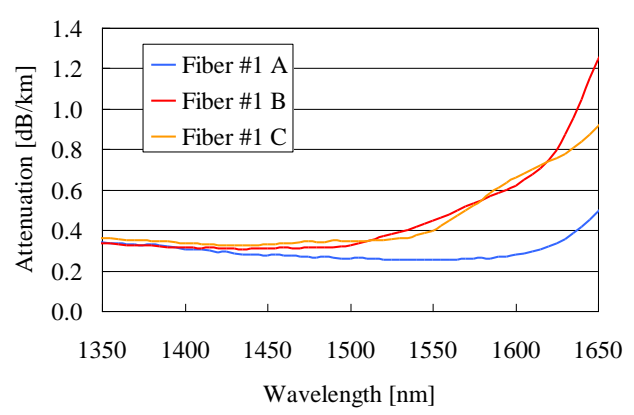

(a)

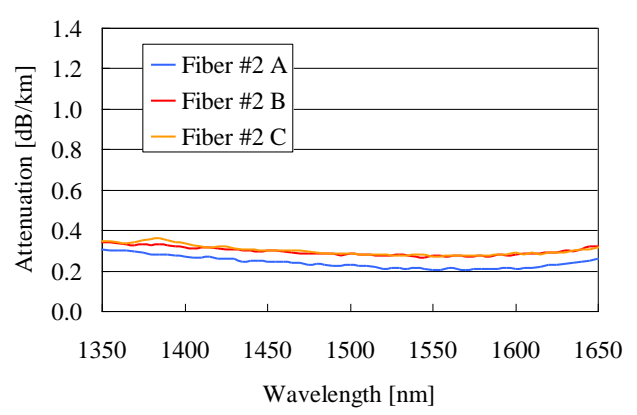

(b)

Fig. 5. Attenuation spectra of fabricated fibers (a) Fiber \#1: $141 \mu \mathrm{m}$, (b) Fiber \#2: $215 \mu \mathrm{m}$.

\section{Improvement of cross-talk properties by optimization of core pitch}

\subsection{Design optimization of core pitch taking the core density into account}

However the attenuation loss was drastically improved by means of sufficiently thick cladding as reported in the section 2, it still needs to be investigated because there was a problem, which is a higher cross-talk. As shown in Table 3, the cross-talks between core A and core B, $\mathrm{C}$ after $2 \mathrm{~km}$ transmission were relatively large (about $-20 \mathrm{~dB}$ ) compared to the simulation results $(<-60 \mathrm{~dB}$ after $100 \mathrm{~km})$. Additionally, the loss increase observed at the longer wavelength region in case of core A of Fiber \#1, which has sufficiently thick cladding, can also be considered due to the higher cross-talk. It was assumed that this high cross-talk was caused by the smaller core diameter difference and smaller core pitch than the designed values. So, we optimized the design taking these factors into account, to improve the optical properties of the large Aeff MCFs.

Table 3. Cross-Talk after 2 km Transmission

\begin{tabular}{|l|l|l|}
\hline \multicolumn{1}{|c|}{ Core No. } & \multicolumn{1}{|c|}{ Fiber \#1 } & \multicolumn{1}{c|}{ Fiber \#2 } \\
\hline B & $-17 \mathrm{~dB}$ & $-21 \mathrm{~dB}$ \\
\hline $\mathrm{C}$ & $-20 \mathrm{~dB}$ & $-18 \mathrm{~dB}$ \\
\hline
\end{tabular}

About the core diameter difference, we tried to solve the problem by setting some margins at the design stage. On the other hand, optimum core pitch was reexamined from the point of views of the cross-talk and a core density. The core density was evaluated by normalized core numbers, which are defined as this expression,

$$
\text { normalized core numbers }=N_{M C F} \frac{S_{S M F}}{S_{M C F}}
$$

which means the effective core numbers of MCFs within an unit area, namely, the area of conventional SMF with $250 \mu \mathrm{m}$ coating diameter (Fig. 6). The coating thickness of the MCFs was set to be the same as the conventional SMF. In Fig. 7, the core pitch dependences of the cross-talk and normalized core numbers are shown. Here, the cross-talk between the identical cores was calculated, which is the most dominant factor of the cross-talk. As a result, it was found that the cross-talk could be suppressed drastically by increasing the core pitch, while the influences to the normalized core numbers were relatively small. So, an enlargement of the core pitch could be regarded as an effective way to improve the cross-talk properties. This time, we set the core pitch as $46 \mu \mathrm{m}$ because the cross-talk could be suppressed by $30 \mathrm{~dB}$ from that of Fiber \#2, which had the core pitch of $40 \mu \mathrm{m}$. 


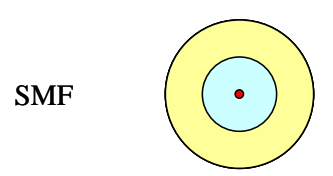

Fiber cross section $S_{\text {SMF }}$

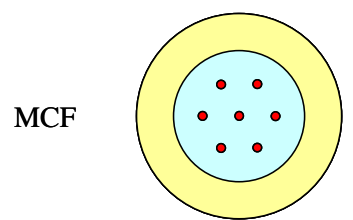

Fiber cross section $\mathrm{S}_{\mathrm{MCF}}$ Core numbers $\mathrm{N}_{\mathrm{MCF}}$

Fig. 6. Definition of the normalized core numbers of MCFs.

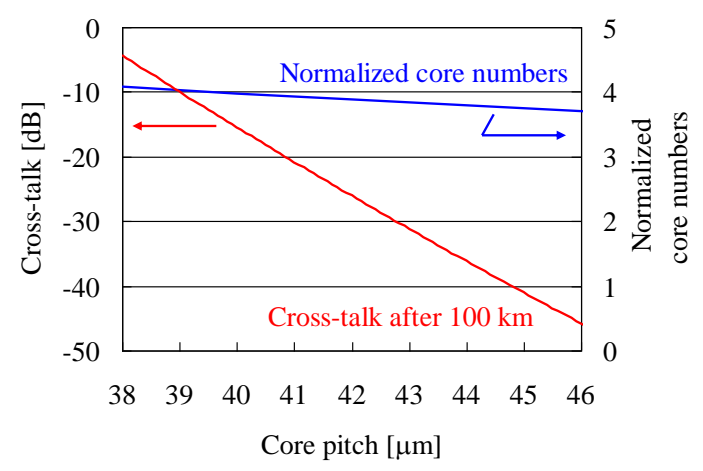

Fig. 7. Core pitch dependence of cross-talk after $100 \mathrm{~km}$ transmission and normalized core numbers.

\subsection{Confirmation of the optimized design by fiber fabrication}

The cross section of the fabricated fiber (Fiber \#3) and the optical properties of the 3 cores with different core diameter (A, B, C) are shown in Fig. 8 and Table 4, respectively. The core $\mathrm{A}$ indicates the center core and core $\mathrm{B}, \mathrm{C}$ indicate the outer cores, again. The cladding diameter of Fiber \#3 was $217 \mu \mathrm{m}$. By the cut-off wavelength measurement results, the core diameter differences were estimated to be almost the same as the design. Other optical properties, such as dispersion and Aeff, were also agreed with the predicted values. The attenuation loss spectra of each core are shown in Fig. 9. The loss values at each core were improved from the previous results (Fiber \#2) because the coating thickness was also optimized to be the same as the conventional SMF $(62.5 \mu \mathrm{m})$, which was thicker than the previous examination $(37.5 \mu \mathrm{m})$. The loss spectrum of the single core fiber with the same core and the same cladding and coating thickness as the outer cores is shown together. The losses of outer cores are slightly higher than that of the center core, but the results are almost same as those of the single core fiber. So, it is supposed that the cladding and coating thickness required for the MCFs can be estimated by the loss properties of the single core fiber.

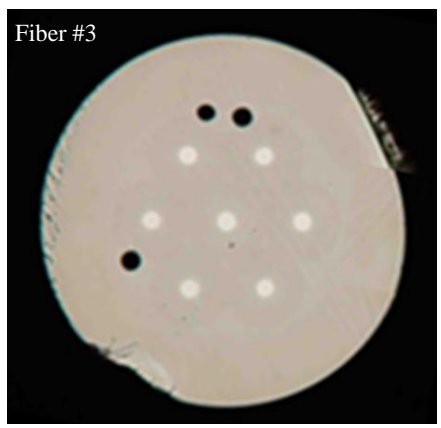

Fig. 8. Cross sections of Fiber \#3.

\#145228 - \$15.00 USD

(C) 2011 OSA
Received 1 Apr 2011; revised 8 May 2011; accepted 9 May 2011; published 13 May 2011 23 May 2011 / Vol. 19, No. 11 / OPTICS EXPRESS 10600 
Table 4. Optical Properties of Fiber \#3 (@1550 nm)

\begin{tabular}{|c|c|c|l|l|l|l|l|}
\hline Fiber & \multirow{2}{*}{$\begin{array}{c}\text { Core } \\
\text { No. }\end{array}$} & Loss & Dispersion & D. Slope & Aeff & $\lambda \mathrm{cc}$ & Bend loss * \\
\cline { 3 - 9 } & \multicolumn{1}{|c|}{$\mathrm{dB} / \mathrm{km}$} & $\mathrm{ps} / \mathrm{nm} / \mathrm{km}$ & $\mathrm{ps} / \mathrm{nm}^{2} / \mathrm{km}$ & $\mu \mathrm{m}^{2}$ & $\mathrm{~nm}$ & $\mathrm{~dB} / \mathrm{m}$ \\
\hline Fiber \#3 & $\mathrm{A}$ & 0.213 & 19.6 & 0.062 & 110 & 1491 & 3.0 \\
\cline { 2 - 9 } & $\mathrm{B}$ & 0.237 & 19.4 & 0.062 & 107 & 1470 & 3.9 \\
\cline { 2 - 9 } & $\mathrm{C}$ & 0.237 & 19.0 & 0.061 & 99 & 1408 & 8.1 \\
\hline
\end{tabular}

* Macro bending loss at $20 \mathrm{~mm}$ diameter.

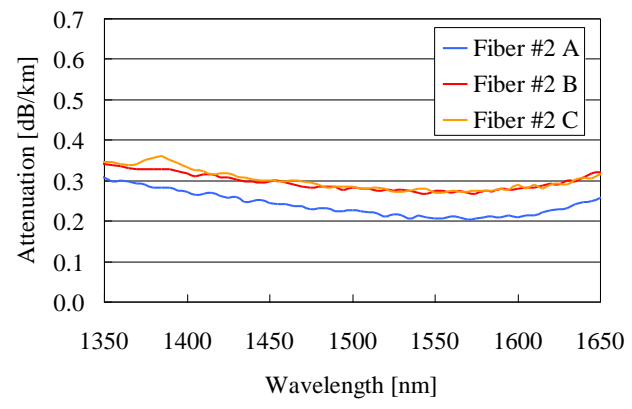

(a)

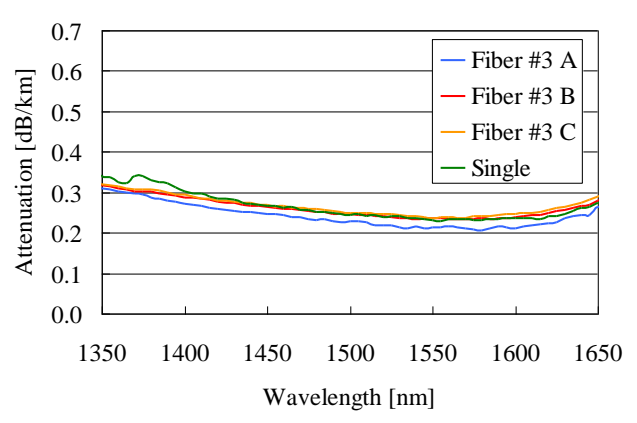

(b)

Fig. 9. Attenuation loss spectra of fabricated fibers (a) Fiber \#2 (re-cited for comparison), (b) Fiber \#3 and single core fiber with the same core diameter, cladding thickness and coating thickness with outer cores.

The measurement results of the cross-talk are shown in Fig. 10. The cross-talk was $-38 \mathrm{~dB}$ after $3.5 \mathrm{~km}$ transmission, and it was improved drastically from the previous result $(-20 \mathrm{~dB}$ after $2 \mathrm{~km}$ ). From the measurement results of the fiber length dependence of the cross-talk, the cross-talk after $100 \mathrm{~km}$ transmission can be estimated as $-33 \mathrm{~dB}$. So, it can be said that the $\mathrm{MCF}$, which can satisfy the cross-talk property required for the long distance transmission, was successfully realized by optimizing the fiber designs.

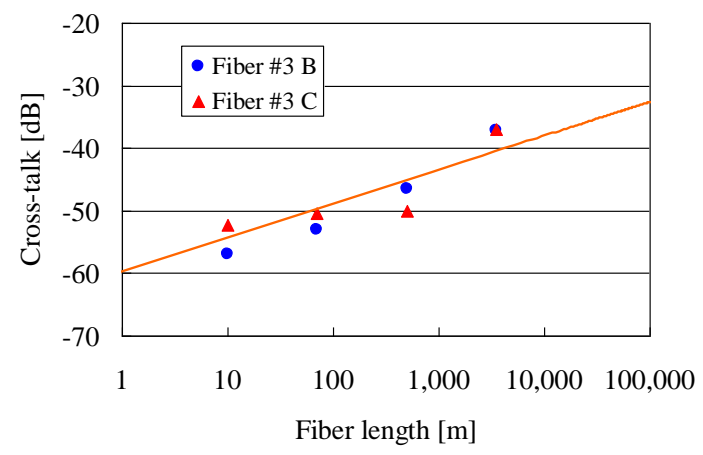

Fig. 10. Fiber length dependence of cross-talk.

\subsection{Estimation of the cross-talk properties by means of coupled power theory}

Though modeling of the cross-talk properties were based on the coupled mode theory in this work, new method for the simulation of cross-talk by applying the coupled power theory has recently been suggested [10]. It was described that more practical cross-talk characteristics can be explained by using the coupled power theory instead of the coupled mode theory, which calculate the interference between cores in case of the ideal situation with the constant core diameter in the fiber length. On the other hand, it is known that the longitudinal 
characteristics of fibers can be estimated by means of the bi-directional optical time-domain reflectometer (OTDR) method [11]. This time, we investigated the longitudinal distribution of core diameter of the fabricated MCFs by using bi-directional OTDR method, and applied the obtained results to the estimation of cross-talk properties based on the coupled power theory. The results of the longitudinal distribution of mode field diameter (MFD) in case of Fiber \#3 are shown in Fig. 11. The noises in the first and last part of figures are caused by the influence of the fresnel reflection at the each side of the fiber. We estimated the longitudinal fluctuations of the core diameters from these MFD distributions and applied them to the calculation of the cross-talk properties based on the coupled power theory in order to reproduce more practical situation than the former case [10] which supposed that the core diameters were changed constantly. The obtained results are shown in Fig. 12. The bending diameter of fiber was set to $280 \mathrm{~mm}$ and the twist rotated in every $20 \mathrm{~m}$ was considered. As a result, it was confirmed that cross-talk properties are successfully calculated by the combination of the bi-directional OTDR method and coupled power theory.

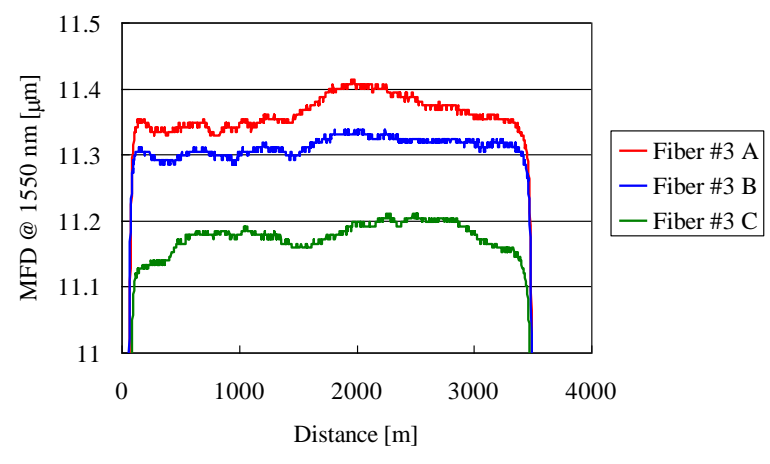

Fig. 11. Longitudinal fluctuation of MFD @ 1550 nm (Fiber \#3). 


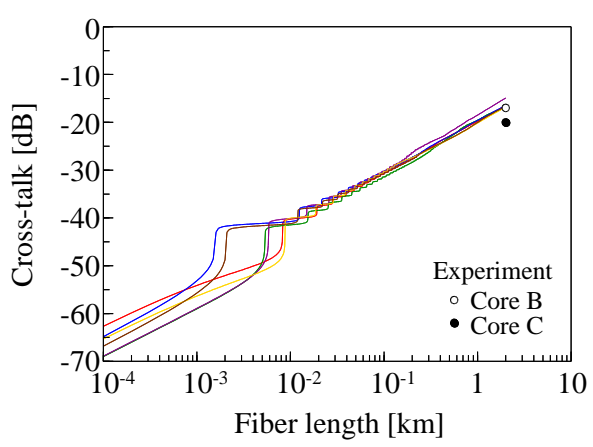

(a)

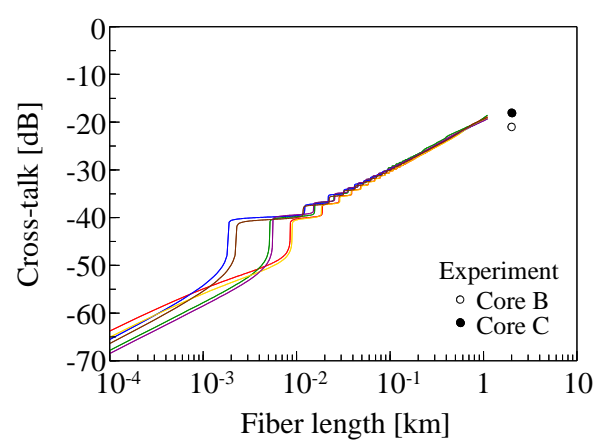

(b)

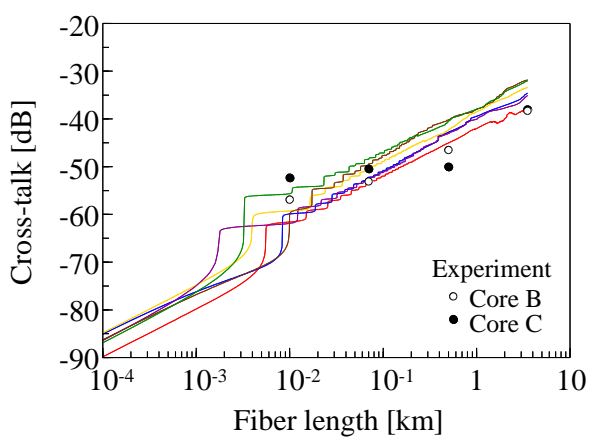

(c)

Fig. 12. Fiber length dependence of cross-talk based on the coupled power theory taking the longitudinal fluctuation of core diameters into account (a) Fiber \#1, (b) Fiber \#2, (c) Fiber \#3.

\section{Conclusion}

To realize large Aeff multi-core fibers, the design strategy to suppress the cross-talk between cores and the micro bending loss were investigated. The MCFs with the large Aeff over 100 $\mu \mathrm{m}^{2}$ and low micro bending loss were successfully fabricated and the obtained results indicate the importance of fiber design to realize large Aeff MCFs including fiber diameters, which largely affect the micro bending loss property. The low cross-talk after long distance transmission was realized by the design optimization and those properties were successfully explained by using the coupled power theory taking the longitudinal fluctuation of core diameter into account.

\section{Acknowledgment}

This research is supported by the National Institute of Information and Communications Technology (NICT), Japan under "Research on Innovative Optical Fiber Technology". 